\title{
Prediction of Treatment Outcome of Chemotherapy Using Perfusion Computed Tomography in Patients with Unresectable Advanced Gastric Cancer
}

\author{
Dong Ho Lee, $M D^{1,2}$, Se Hyung Kim, $M D^{1,2}$, Sang Min Lee, $M D^{3}$, Joon Koo Han, $M D^{1,2,4}$ \\ ${ }^{1}$ Department of Radiology, Seoul National University Hospital, Seoul, Korea; ${ }^{2}$ Department of Radiology, Seoul National University College of

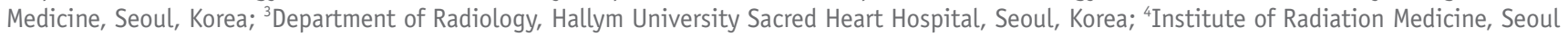 \\ National University Medical Research Center, Seoul, Korea
}

Objective: To evaluate whether data acquired from perfusion computed tomography (PCT) parameters can aid in the prediction of treatment outcome after palliative chemotherapy in patients with unresectable advanced gastric cancer (AGC). Materials and Methods: Twenty-one patients with unresectable AGCs, who underwent both PCT and palliative chemotherapy, were prospectively included. Treatment response was assessed according to Response Evaluation Criteria in Solid Tumors version 1.1 (i.e., patients who achieved complete or partial response were classified as responders). The relationship between tumor response and PCT parameters was evaluated using the Mann-Whitney test and receiver operating characteristic analysis. One-year survival was estimated using the Kaplan-Meier method.

Results: After chemotherapy, six patients exhibited partial response and were allocated to the responder group while the remaining 15 patients were allocated to the non-responder group. Permeability surface (PS) value was shown to be significantly different between the responder and non-responder groups $(51.0 \mathrm{~mL} / 100 \mathrm{~g} / \mathrm{min}$ vs. $23.4 \mathrm{~mL} / 100 \mathrm{~g} / \mathrm{min}$, respectively; $p=0.002$ ), whereas other PCT parameters did not demonstrate a significant difference. The area under the curve for prediction in responders was $0.911(p=0.004)$ for PS value, with a sensitivity of $100 \%(6 / 6)$ and specificity of $80 \%$ $(12 / 15)$ at a cut-off value of $29.7 \mathrm{~mL} / 100 \mathrm{~g} / \mathrm{min}$. One-year survival in nine patients with PS value $>29.7 \mathrm{~mL} / 100 \mathrm{~g} / \mathrm{min}$ was $66.7 \%$, which was significantly higher than that in the 12 patients $(33.3 \%)$ with PS value $\leq 29.7 \mathrm{~mL} / 100 \mathrm{~g} / \mathrm{min}(p=0.019)$. Conclusion: Perfusion parameter data acquired from PCT demonstrated predictive value for treatment outcome after palliative chemotherapy, reflected by the significantly higher PS value in the responder group compared with the non-responder group. Keywords: Stomach; Functional imaging; Reproducibility; Survival; Permeability

\section{INTRODUCTION}

Gastric cancer is one of the most common malignant tumors and the second leading cause of cancer-related death worldwide $(1,2)$. Among the various treatment options for patients with gastric cancer, surgical resection is the only known curative treatment able to achieve longterm survival (3). Unfortunately, however, surgical resection cannot be performed in a substantial proportion of gastric cancer patients because more than two-thirds exhibit unresectable disease owing to the presence of distant metastasis and/or invasion to adjacent organs such as the pancreas (2). Thus, in patients with unresectable advanced gastric cancer (AGC), palliative chemotherapy, which has demonstrated the potential to improve both patient survival and quality of life, has been the standard treatment method

Received May 9, 2018; accepted after revision October 3, 2018.

This study was supported by the Basic Science Research Program through the National Research Foundation of Korea (NRF) funded by the Ministry of Science, ICT \& Future Planning (2016R1A2B4007762).

Corresponding author: Se Hyung Kim, MD, Department of Radiology, Seoul National University Hospital and Seoul National University College of Medicine, 101 Daehak-ro, Jongno-gu, Seoul 03080, Korea.

- Tel: (822) 2072-2057 • Fax: (822) 743-6385• E-mail: shkim7071@gmail.com

This is an Open Access article distributed under the terms of the Creative Commons Attribution Non-Commercial License (https:// creativecommons.org/licenses/by-nc/4.0) which permits unrestricted non-commercial use, distribution, and reproduction in any medium, provided the original work is properly cited. 
$(4,5)$. However, the reported response rates of unresectable AGCs to palliative chemotherapy has ranged only between $40 \%$ and $70 \%$. Therefore, earlier prediction of treatment response to palliative chemotherapy would be clinically important, and enable more appropriate management planning including the early termination of ineffective chemotherapy regimens and replacing them with more effective alternatives.

Traditionally, the treatment response to systemic chemotherapy in patients with malignant diseases, such as AGC, has been evaluated using morphological sizebased criteria, for which the Response Evaluation Criteria in Solid Tumors (RECIST) version 1.1 has been the most widely used method (6). However, it is also well-known that after systemic chemotherapy, perfusion and/or biological changes can develop within the tumor before any change in tumor size. Therefore, to overcome this limitation of current morphological size-based criteria, several functional imaging approaches, such as multiparametric magnetic resonance imaging (MRI), positron emission tomography, and perfusion imaging using either $\mathrm{CT}$ or MR have been introduced (7-12). Among them, perfusion CT (PCT) has emerged as a promising functional imaging modality able to evaluate tissue perfusion characteristics in acute stroke and oncological patients (13-16). In fact, several studies have reported that obtaining perfusion parameter data from PCT in gastric cancer patients may be feasible, and that several perfusion parameters calculated from this PCT dataset may provide information regarding vascularity as well as the neo-angiogenesis of gastric cancers (17-20). Our group also recently reported that the permeability surface (PS) and mean transit time (MTT) values of gastric cancer can help in the preoperative imaging diagnosis of poorly cohesive carcinoma (PCC)-type AGCs, which generally have a worse prognosis compared with other histological subtypes of gastric cancer, even after curative resection (21). However, to date, the potential utility of PCT in predicting treatment response after systemic chemotherapy in patients with unresectable AGCs has not been investigated. Therefore, the purpose of this study was to prospectively evaluate whether perfusion parameter data acquired from the PCT dataset can help predict treatment response, including one-year survival, in patients with unresectable AGC.

\section{MATERIALS AND METHODS}

The Institutional Review Board of the authors' hospital approved this prospective study, and informed written informed consent was obtained from all patients.

\section{Patients}

From October 2015 to September 2016, 26 patients who met the following inclusion criteria were prospectively enrolled in this study: histopathologically proven gastric cancers diagnosed through endoscopic biopsy; preoperative staging workup including multi-detector contrast-enhanced CT (MDCT) scans indicating unresectable disease owing to either the presence of distant metastasis or invasion of adjacent organs including the pancreas, liver, diaphragm, or colon; and palliative chemotherapy planned to control the unresectable AGC. PCT was performed in all 26 patients, among whom, five were excluded for the following reasons: refusal of palliative chemotherapy $(n=3)$; failure to start palliative chemotherapy due to the rapid progression of AGC with deterioration in performance status $(n=1)$; and failure to complete the scheduled chemotherapy due to intolerable toxicity (i.e., severe neutropenia with fever) (n $=1$ ). Therefore, 21 patients (13 male, eight female; mean [ \pm standard deviation] age, $53.7 \pm 14.3$ years [age range, 31-83 years]) ultimately comprised the study population (Fig. 1). The reasons for un-resectability were the presence of distant metastasis in 19 patients and invasion of the pancreas by AGC in the remaining two patients. In the 19 patients with distant metastasis, the location of distant metastasis on imaging examination included the liver $(n=$ $8)$, peritoneum $(n=5)$, retroperitoneal lymph node $(n=4)$,

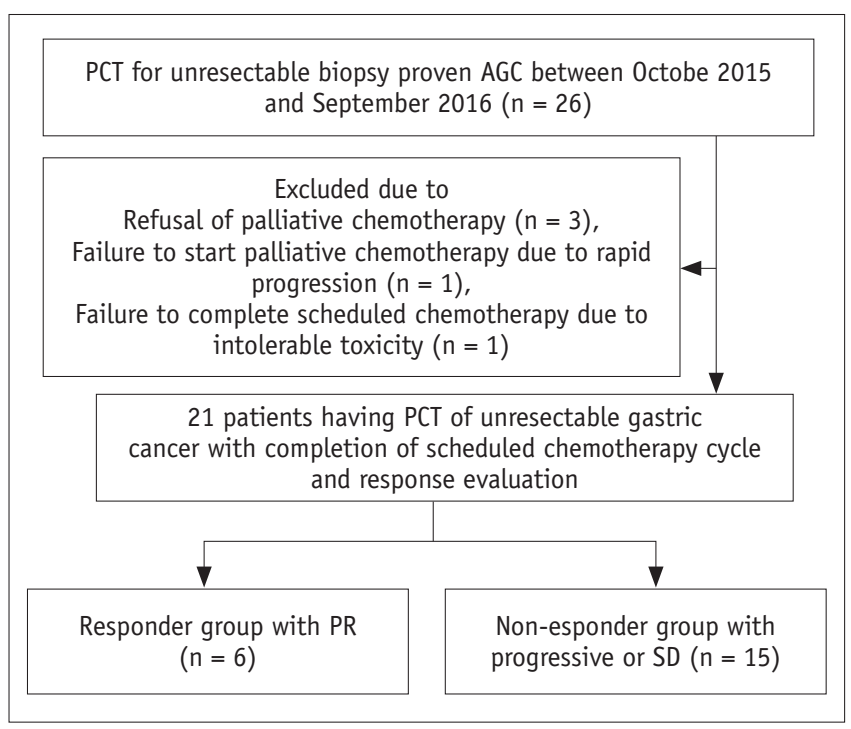

Fig. 1. Flow chart illustrating patient enrollment process. $A G C=$ advanced gastric cancer, $\mathrm{PCT}=$ perfusion $\mathrm{CT}, \mathrm{PR}=$ partial response, $\mathrm{SD}=$ stable disease 
lung $(n=1)$, and ovary $(n=1)$. All eight patients with liver metastasis, one patient with lung metastasis, and two with peritoneal seeding were diagnosed through histopathological examination using specimens obtained through percutaneous biopsy (liver metastases [ $n=8]$ and lung metastasis [ $\mathrm{n}=$ 1]) or diagnostic laparoscopy (peritoneal seeding $[n=2]$ ). The remaining three patients with peritoneal seeding, one with ovarian metastasis, and all four patients with retroperitoneal lymph node metastases were diagnosed clinically. The histological subtypes of AGC included: moderately-differentiated adenocarcinoma $(n=5)$; poorlydifferentiated adenocarcinoma $(n=10)$; and PCC $(n=6)$.

\section{PCT Acquisition}

All PCT examinations were performed using an MDCT scanner (Definition; Siemens Healthineers, Erlangen, Germany). All participating patients fasted for at least 6 hours before PCT acquisition, and each patient drank 500$1000 \mathrm{~mL}$ of water before the PCT examination to distend the stomach for better delineation of the gastric cancer lesion(s) (12, 17, 20, 22). Hyoscine butylbromide (10 mg; Buscopan, Boehringer Ingelheim Korea, Seoul, Korea) was also injected intravenously 5 minutes before PCT acquisition if not contraindicated so as to minimize gastric movement during the examination, which could cause motion-related artifacts $(12,21)$. To minimize respiratory motion-related artifacts during the PCT examination, patients were asked to breathe as calmly as possible. For PCT acquisition, noncontrast images were initially acquired before contrast injection from the liver dome to the lower margin of the right kidney, and the exact location of the gastric cancer was identified. Thereafter, $40 \mathrm{~mL}$ of a nonionic iodinated contrast agent (350 mg I/mL; iobitridol, Xenetix 350, Guerbet, Aulnay-sous-Bois, France) was injected at a rate of $5 \mathrm{~mL} / \mathrm{s}$ through an 18-gauge catheter placed into an antecubital vein, followed by a saline flush of $50 \mathrm{~mL}$ at the same rate using a power injector. PCT acquisition was started 6 seconds after contrast injection because there can be an approximately 10 seconds time interval between contrast injection and contrast arrival to the aorta (21). PCT scans were performed using the following parameters: scan range, $8 \mathrm{~cm}$ (according to the location of the AGC); gantry rotation time, 0.4 seconds; effective tube current, $100 \mathrm{mAs}$ at $80 \mathrm{kVp}$ power; reconstruction section thickness, $3 \mathrm{~mm}$; cycle time, 3.6 seconds; and number of scans, 15, which lasted for 60 seconds. Mean dose length product of PCT in the 21 patients was $975.8 \pm 261.9 \mathrm{mGy} \cdot \mathrm{cm}$ (range,
675-1548 $\mathrm{mGy} \cdot \mathrm{cm})$. The estimated mean effective radiation dose was $14.6 \pm 3.93 \mathrm{mSv}$ with a conversion factor of 0.015 $\mathrm{mSv} / \mathrm{mGy} \cdot \mathrm{cm}(21,23)$.

\section{PCT Analysis}

For the analysis of PCT data, a dedicated perfusion software program (VPCT body, Siemens Healthineers) using the maximum slope and delayed Patlak analysis models was used. PCT analyses of AGCs were performed by two different abdominal radiologists (with 9 and 5 years' experience in abdominal perfusion imaging, respectively) who were aware that the patients had AGCs and were treated with systemic chemotherapy, but were blinded to the response of AGC to chemotherapy. First, the reconstructed images were transferred to a workstation where the perfusion software program was installed. Thereafter, a motion correction algorithm using the non-rigid registration method was applied for each PCT examination so as to minimize analysis errors owing to motion during the PCT acquisition. Thereafter, the aorta was chosen as the input artery with regions of interest (ROIs) placed on the abdominal aorta in the representative axial plane. ROIs were manually drawn for the AGC lesion to be as large as possible on the representative axial plane, with special care taken not to include perigastric fat, or intragastric air or water (21). The mean area of the ROIs drawn on the tumor was $839.3 \pm$ $511.2 \mathrm{~mm}^{2}$ (range, 261.2-1954.1 $\mathrm{mm}^{2}$ ). Finally, parametric perfusion maps were generated using a high-resolution, pixel-by-pixel calculation method which automatically calculated the ROI values of the following perfusion parameters: blood flow (BF) $(\mathrm{mL} / 100 \mathrm{~g} / \mathrm{min})$; blood volume (BV) (mL/100 g); MTT (seconds); time to peak (TTP) (seconds); and PS ( $\mathrm{mL} / 100 \mathrm{~g} / \mathrm{min})$. These resultant perfusion parameters for $\mathrm{AGC}$ were recorded for further analysis.

\section{Palliative Chemotherapy and Response Evaluation}

After PCT examination, all patients underwent palliative chemotherapy using one of the three following regimens: XELOX, which is a combination of capecitabine (Xeloda ${ }^{\circledR}$ ) and cisplatin and oxaliplatin in 10 patients; FOLFOX, a combination of infusional 5-fluorouracil (5-FU), leucovorin and oxaliplatin in 7 patients; and XP, a combination of capecitabine $\left(\right.$ Xeloda $\left.^{\circledR}\right)$ and cisplatin in 4 patients. Treatments were repeated every two or three weeks using the same regimen until disease progression and/ or development of intolerable severe toxicity. Treatment response to chemotherapy was evaluated by one abdominal 
radiologist (with 9 years' experience in clinical radiology) using RECIST version 1.1 (6). Follow-up contrast-enhanced abdominal CT was performed and each patient was evaluated to determine treatment response. The mean interval between the start of chemotherapy and response evaluation was $61.3 \pm 18.1$ days (range, $23-90$ days). After determination of the response category of each patient, patients were classified into two groups: the responder group for patients in whom complete response (CR) or partial response (PR) was observed; and the non-responder group for patients who were determined to have a stable disease (SD) or progressive disease (PD).

\section{Statistical Analysis}

To compare baseline characteristics, distribution of histological subtypes, chemotherapy regimens, as well as PCT perfusion parameters between those in the responder and non-responder groups, Fisher's exact test was used for categorical variables and the Mann-Whitney $\mathrm{U}$ test for continuous variables. When significant differences in perfusion parameters were found between the two groups, receiver operating characteristic (ROC) analysis was performed. The area under the curve (AUC) was estimated using ROC analysis with the determination of the optimal cut-off value to calculate sensitivity and specificity. In addition, the Mann-Whitney $U$ test was used to compare PCT parameters between PCC and non-PCC type of AGCs.

To assess the measurement reproducibility of PCT perfusion parameters of AGC between the two radiologists, intraclass correlation coefficients (ICCs) with two-tailed $p$ values were used. An ICC value $>0.81$ was considered to represent almost perfect agreement, and values of 0.61 $0.80,0.41-0.60$, and $0.21-0.40$, to represent substantial, moderate, and fair agreement, respectively (12). An ICC value $<0.20$ indicated slight agreement. In addition, the Bland-Altman method was used to calculate $95 \%$ limits of agreement for each perfusion parameter of AGC obtained from each radiologist.

To compare the survival rate after palliative chemotherapy, the study population was divided into two groups according to the cut-off PCT value determined by the ROC analysis. Patient survival after palliative chemotherapy for AGC was defined as the interval between start of treatment and patient death or the last follow-up visit before December 31, 2017, and estimated using the Kaplan-Meier method. A log-rank test was used to compare the survival rate between the patient groups. The Cox proportional hazard regression model was used to identify the significant determinant factors for survival after palliative chemotherapy for unresectable AGC. All statistical analyses were performed using SPSS version 22 (IBM Corp., Armonk, NY, USA) and MedCalc software version 12.2.1.0 (Mariakerke, Belgium); $p$ $<0.05$ was considered to indicate statistical significance.

\section{RESULTS}

Technical Success and Reproducibility of Measurements

Acquisition and analysis of PCT were possible in all 21 patients. ICC values of perfusion parameters between the two radiologists were 0.828 for $\mathrm{BF}, 0.869$ for $\mathrm{BV}, 0.787$ for TTP, 0.442 for MTT, and 0.843 for PS, indicating moderate to almost perfect agreement. The $95 \%$ limits of agreement assessed using the Bland-Altman plots were: $-56.5-45.3 \%$ for BF; -55.7-39.9\% for BV; $-13.0-14.8 \%$ for TTP; $-65.9-$ $76.3 \%$ for MTT; and $-82.4-103.4 \%$ for PS.

\section{Response Evaluation after Chemotherapy}

After chemotherapy, PR was achieved in six patients, $S D$ in nine, and PD in six. No patients achieved CR after chemotherapy. Therefore, 6 patients with PR were allocated to the responder group (6/21 [28.6\%]) and 15 (6 with PD and 9 with SD) to the non-responder group. There were no significant differences in chemotherapy regimens between the two groups $(p=0.983)$. In addition, there were no significant differences in all PCT parameters including PS and MTT between PCC and non-PCC histological subtypes ( $p$ $>0.05$ for all PCT parameters).

\section{Predictors of Treatment Response}

Predictors of treatment response to systemic chemotherapy in patients with unresectable AGCs are summarized in Table 1. Mean PS value $(51.0 \mathrm{~mL} / 100 \mathrm{~g} / \mathrm{min})$ of AGCs in the responder group was significantly higher than in the non-responder group $(23.4 \mathrm{~mL} / 100 \mathrm{~g} / \mathrm{min})(p$ $=0.002$ ) (Figs. 2-4). The other perfusion parameters were not revealed to be significantly different between the two groups. Histological subtype (PCC versus non-PCC) and differentiation (moderately differentiated versus poorly differentiated) of gastric cancer also did not significantly differ between the groups.

ROC analysis revealed that the AUC for PS was 0.911 (95\% confidence interval [CI] $0.780-1.000 ; p<0.001$ ) (Fig. 5). A sensitivity of $100 \%(6 / 6)$ and a specificity of $80 \%(12 / 15)$ for chemotherapy response were achieved at a cut-off value 
Table 1. Predictors of Treatment Response to Chemotherapy

\begin{tabular}{lccc}
\hline \multicolumn{1}{c}{ Parameters } & Responders $(\mathrm{n}=6)$ & Non-Responders $(\mathrm{n}=15)$ & $P^{*}$ \\
\hline PCT & & & $51.4 \pm 17.6$ \\
BF (mL/100 g/min) & $46.5 \pm 14.7$ & $11.0 \pm 3.7$ & 0.569 \\
BV (mL/100 g) & $11.2 \pm 3.8$ & $15.0 \pm 1.5$ & 0.970 \\
TTP (seconds) & $16.0 \pm 1.7$ & $10.3 \pm 3.5$ & 0.267 \\
MTT (seconds) & $13.3 \pm 3.8$ & $23.4 \pm 8.4$ & 0.095 \\
PS (mL/100 g/min) & $51.0 \pm 29.5$ & $51.3 \pm 16.2$ & 0.002 \\
Age (years) & $59.7 \pm 5.5$ & $8: 7$ & 0.080 \\
Sex (male:female) & $5: 1$ & $5: 10$ & 0.201 \\
Histologic subtype (PCC:non-PCC) & $1: 5$ & $4: 11$ & 0.623 \\
Differentiation (M/D:P/D) & $1: 5$ & 1.000 \\
\hline
\end{tabular}

Data are means \pm standard deviation. * $p$ values were determined by Fisher's exact test for categorical variables and Mann-Whitney $U$ test for continuous variables. $\mathrm{BF}=$ blood flow, $\mathrm{BV}=$ blood volume, $\mathrm{M} / \mathrm{D}=$ moderately-differentiated, $\mathrm{MTT}=$ mean transit time, $\mathrm{PCC}=\mathrm{poorly}$ cohesive carcinoma, $\mathrm{PCT}=$ perfusion $\mathrm{CT}, \mathrm{P} / \mathrm{D}=$ poorly-differentiated, $\mathrm{PS}=$ permeability surface, $\mathrm{TTP}=$ time to peak

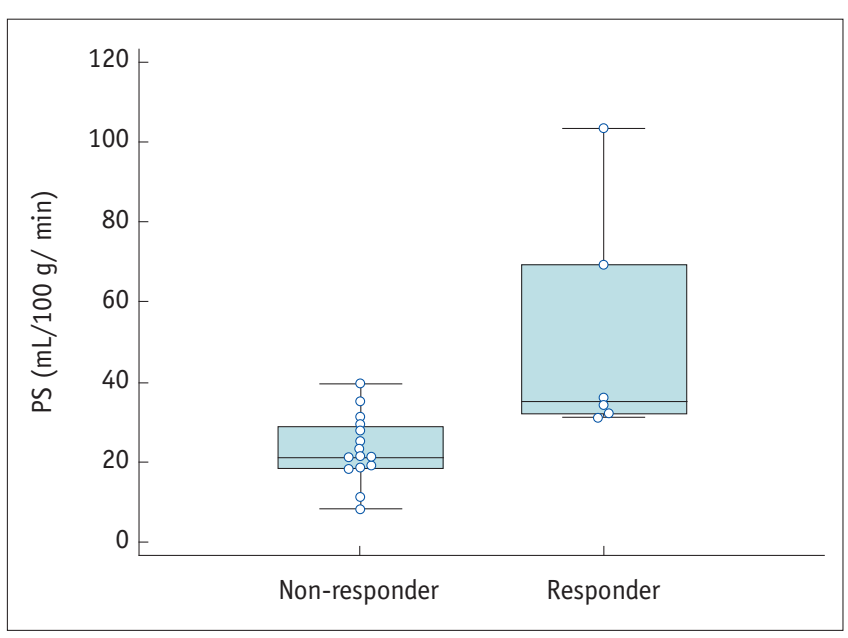

Fig. 2. PS value of each patient group. Mean PS value $(51.0$ $\mathrm{mL} / 100 \mathrm{~g} / \mathrm{min}$ ) of AGCs in responder group was significantly higher than in non-responder group $(23.4 \mathrm{~mL} / 100 \mathrm{~g} / \mathrm{min})(p=0.002)$. All values appear as circles. PS $=$ permeability surface

of $29.7 \mathrm{~mL} / 100 \mathrm{~g} / \mathrm{min}$ for the PS value. The corresponding positive predictive value was $66.7 \%(6 / 9)$ and the negative predictive value was $100 \%(12 / 12)$.

\section{Survival Outcome}

After a mean and median follow-up of $12.4 \pm 7.8$ months and 10.0 months, respectively, 18 patients died due to the progression of $\mathrm{AGC}$, and the remaining three patients were still alive at the end of the study period. The estimated 1-year survival after palliative chemotherapy for AGC in all 21 patients was $47.6 \%$. The estimated 1-year survival in nine patients with $>29.7 \mathrm{~mL} / 100 \mathrm{~g} / \mathrm{min}$ of PS value was $66.7 \%$ and was significantly higher than in the 12 patients with PS value $\leq 29.7 \mathrm{~mL} / 100 \mathrm{~g} / \mathrm{min}(33.3 \%)(p=$ 0.019) (Fig. 6). Cox proportional hazard regression analysis revealed that PS value of $A G C>29.7 \mathrm{~mL} / 100 \mathrm{~g} / \mathrm{min}$ was the only significant predictive factor for better survival (hazard ratio 0.320 [95\% CI 0.11-0.90]; $p=0.030$ ) (Table 2). Other parameters, such as chemotherapy regimen, did not significantly affect patient survival.

\section{DISCUSSION}

In our study, perfusion parameter data were successfully acquired from PCT in all patients with unresectable AGCs, with moderate to almost perfect agreement between the two radiologists. In addition, the mean PS value (51.0 $\mathrm{mL} / 100 \mathrm{~g} / \mathrm{min}$ ) of AGCs in the responder group was significantly higher than in the non-responder group $(23.4 \mathrm{~mL} / 100 \mathrm{~g} / \mathrm{min})(p=0.002)$. This suggests that PCT parameters specifically, the PS value may be used as a predictive marker for the treatment response to systemic chemotherapy. Furthermore, PS value $>29.7 \mathrm{~mL} / 100 \mathrm{~g} / \mathrm{min}$ was the only significant predictive factor for better survival after palliative chemotherapy, probably due to better response to chemotherapy in this patient group. According to our results, the difference in mean PS values between the responder and non-responder groups was $27.6 \mathrm{~mL} / 100 \mathrm{~g} /$ $\min$, a difference of $118 \%$. Considering that the $95 \%$ limits of agreement for the PS value between the radiologists was $-82.4-103.4 \%$, and that this difference of $118 \%$ was beyond the $95 \%$ limits of agreement, our results can be interpreted with confidence. Recently, PCT has emerged as an attractive functional imaging modality that can provide information regarding both the vascularity and neoangiogenesis process of tumors in oncologic fields (13-16). It has also been shown to provide prognostic information 
regarding the treatment response to systemic chemotherapy (24). Therefore, our study results reconfirm the value of PCT in providing clinically relevant information in patients with unresectable AGCs.

By definition, PS values can reflect the contrast media transmission rate from the intravascular space to the

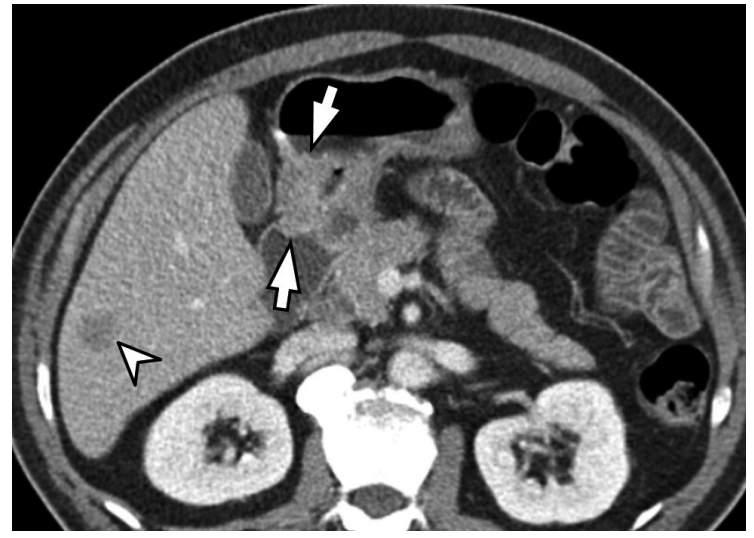

A

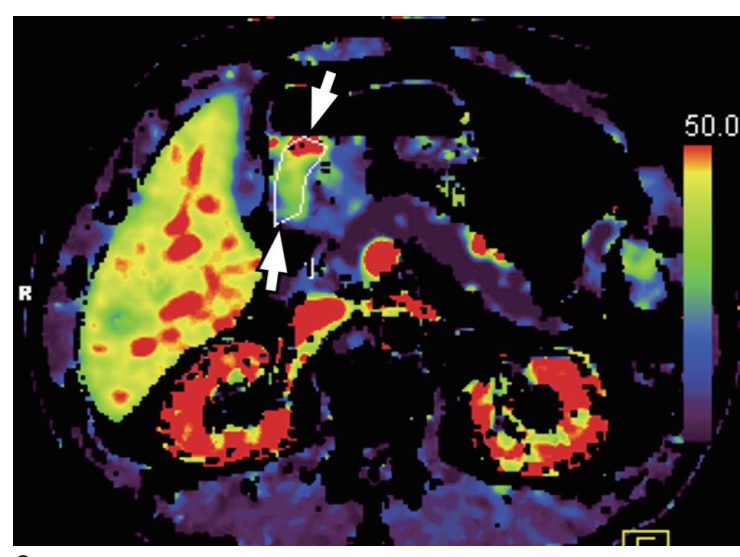

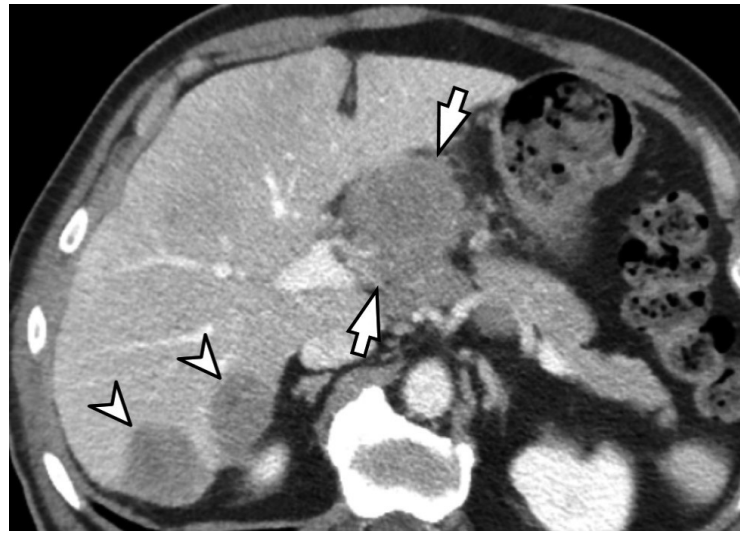

B

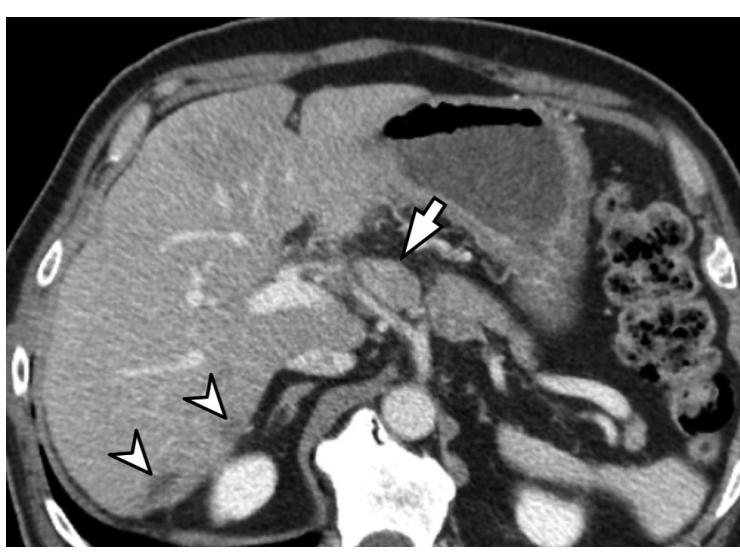

D

Fig. 3. 55-year-old man with histologically confirmed gastric cancer and liver metastasis.

A, B. Contrast-enhanced axial CT images acquired during portal phase reveal marked enhancing wall thickening in antrum of stomach (arrows in A), suggesting AGC. Liver metastasis (arrowhead in A) is also noted. Other metastatic nodules in liver (arrowheads in B) and lymph nodes (arrows in B) are also apparent. C. On parametric perfusion map, PS value of primary gastric cancer was $69.32 \mathrm{~mL} / 100 \mathrm{~g} / \mathrm{min}$, which is higher than cut-off value of $29.7 \mathrm{~mL} / 100 \mathrm{~g} / \mathrm{min}$ (arrows). D. Follow-up axial CT image obtained after two cycles of fluorouracil and oxaliplatin (i.e., FOLFOX) chemotherapy reveals marked decrease in size for both hepatic (arrowheads) and lymph node (arrow) metastases, indicating PR. CT = computed tomography

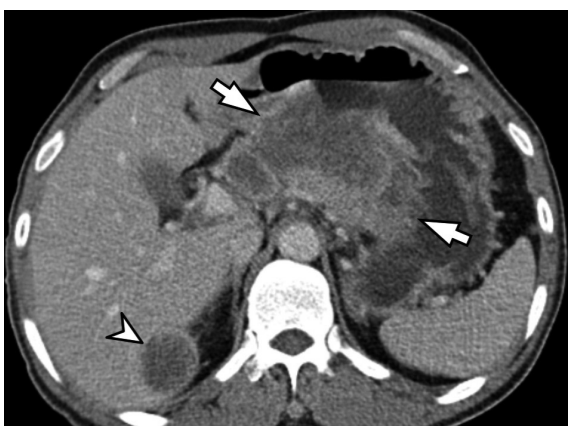

A

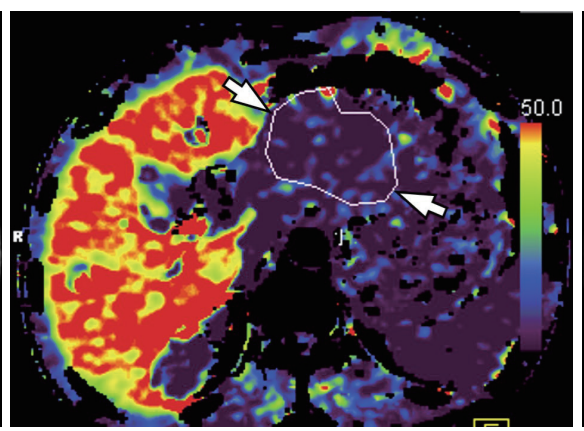

B

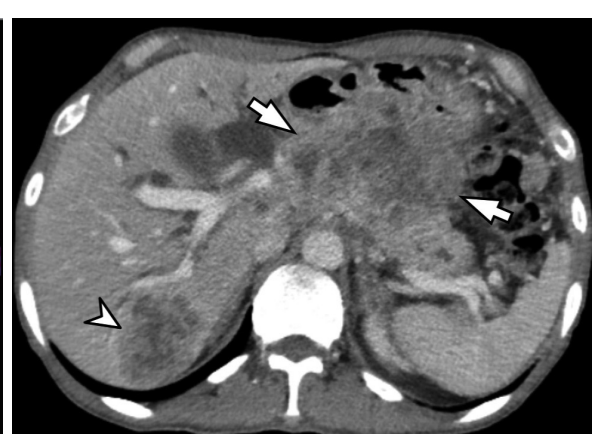

C

Fig. 4. 56-year-old man with histologically confirmed gastric cancer and liver metastasis.

A. Contrast-enhanced axial CT image acquired during portal phase demonstrates heterogeneously enhancing mass in lesser curvature side of gastric body (arrows), suggesting AGC. $3.5 \mathrm{~cm}$ metastasis is also noted in segment VI of liver (arrowhead). B. On parametric perfusion map, PS value of gastric cancer (arrows) was $11.30 \mathrm{~mL} / 100 \mathrm{~g} / \mathrm{min}$, which is lower than cut-off value of $29.7 \mathrm{~mL} / 100 \mathrm{~g} / \mathrm{min}$. C. On follow-up axial CT image acquired after two cycles of capecitabine and cisplatin chemotherapy, liver metastasis demonstrates increase in size from $3.5 \mathrm{~cm}$ to $4.8 \mathrm{~cm}$ (arrowhead), indicating PD. Primary stomach mass (arrows) did not exhibit significant size change after chemotherapy. $\mathrm{PD}=$ progressive disease 
extravascular and extracellular space. Therefore, it can provide information regarding the integrity of endothelial cells and vascular permeability (17). In addition, previous studies have reported that malignant tumors often have higher PS values than normal tissue, owing to the large number of fenestrations, and discontinuous or absent basement membranes in the tumor capillaries and neovasculature $(25,26)$. In specific application, Bellomi et al. (27) reported that PS values of rectal cancer were significantly higher than that of the normal rectal wall. Considering this theoretical background, higher PS values can be considered to reflect more permeable and leaky tumor vasculature. Therefore, this more permeable tumor vasculature may provide better access to chemotherapeutic agents, which in turn may lead to a more favorable response to systemic chemotherapy $(12,28)$. In fact, according to

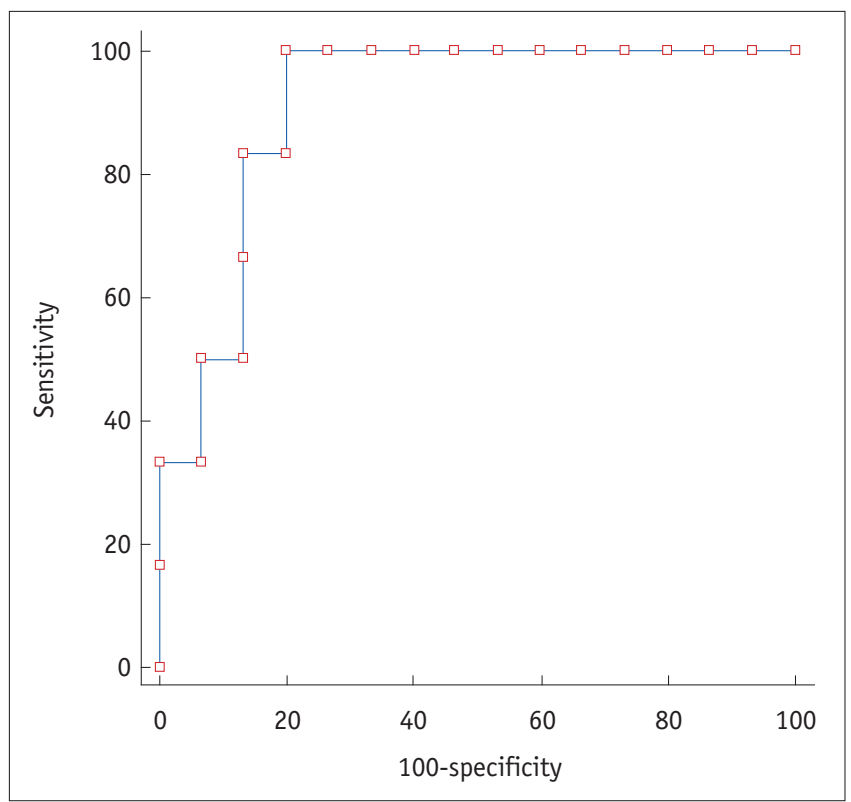

Fig. 5. Results of ROC analysis. ROC analysis revealed that area under curve for PS value was 0.911 (95\% CI $0.780-1.000 ; p<0.001)$. When cut-off value was set at $29.7 \mathrm{~mL} / 100 \mathrm{~g} / \mathrm{min}$ (arrow), sensitivity of $100 \%(6 / 6)$ and specificity of $80 \%(12 / 15)$ were achieved. $\mathrm{CI}=$ confidence interval, ROC $=$ receiver operating characteristic curve a recent article by Kruk-Bachonko et al. (22), neoadjuvant therapy was shown to be more effective in gastric cancer patients with higher PS values. Our study findings also correlate well with this recent result. The potential importance of vascular permeability in the prediction of treatment response to chemotherapy in patients with malignant tumors has also been reported in perfusion MRI. According to Lim et al. (29), locally advanced rectal cancers with high $\mathrm{K}^{\text {trans }}$ values, which also reflects vascular permeability, responded favorably to neoadjuvant chemoradiation therapy. Our group also recently reported that $K^{\text {trans }}$ values of AGCs obtained from multi-parametric MRI, including perfusion MRI, was a significant predictive factor for treatment response as $\mathrm{K}^{\text {trans }}$ values in respondergroup patients were significantly higher than that of nonresponder-group patients (12). Considering the results of these previous studies, as well as our study, we believe that the PS value obtained from PCT could be useful in the prediction of treatment response in patients with AGCs

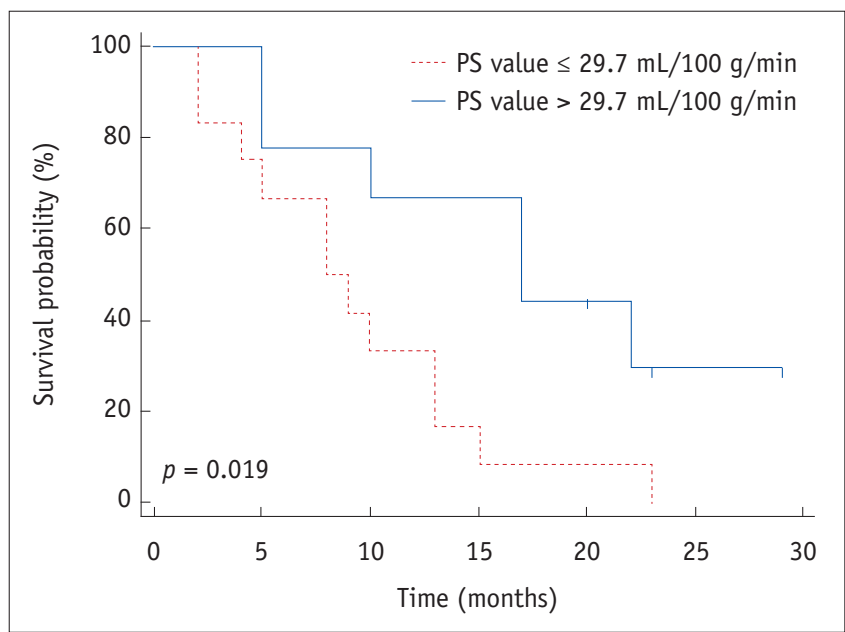

Fig. 6. Patient survival estimation after palliative chemotherapy for AGC. Kaplan-Meier plot reveals that survival rate in 9 patients with PS value $>29.7 \mathrm{~mL} / 100 \mathrm{~g} / \mathrm{min}$ was significantly better than that in 12 patients with PS value $\leq 29.7 \mathrm{~mL} / 100 \mathrm{~g} / \mathrm{min}$ $(p=0.019)$.

Table 2. Cox Survival Analysis for Prediction of Patient Survival

\begin{tabular}{|c|c|c|c|c|c|c|}
\hline \multirow{2}{*}{ Characteristic } & \multicolumn{3}{|c|}{ Univariate } & \multicolumn{3}{|c|}{ Multivariate } \\
\hline & $\mathrm{HR}$ & $95 \% \mathrm{CI}$ & $P$ & $\mathrm{HR}$ & $95 \% \mathrm{CI}$ & $P$ \\
\hline Age (per 1 year) & 0.99 & $0.95-1.02$ & 0.400 & & & \\
\hline Sex & 1.78 & $0.67-4.73$ & 0.246 & & & \\
\hline Histology (PCC vs. non-PCC) & 1.20 & $0.42-3.38$ & 0.734 & & & \\
\hline Differentiation (M/D vs. P/D) & 1.18 & $0.39-3.60$ & 0.768 & & & \\
\hline Chemotherapy regimen & 0.95 & $0.53-1.70$ & 0.861 & & & \\
\hline $\mathrm{PS}>29.7 \mathrm{~mL} / 100 \mathrm{~g} / \mathrm{min}$ & 0.32 & $0.11-0.90$ & 0.030 & 0.32 & $0.11-0.90$ & 0.030 \\
\hline
\end{tabular}

$\mathrm{CI}=$ confidence interval, $\mathrm{HR}=$ hazard risk 
treated with systemic chemotherapy.

In our study, all CT perfusion parameters, including MTT and PS of PCC type of AGC, were not significantly different from those of non-PCC type of AGC. This result was somewhat different from the result of our previous investigation (21), in that PS and MTT values of PCC type of AGC were significantly higher than those of non-PCC type AGC. We believe that this difference can, in large part, be explained by the difference in reference standard between two studies: endoscopic biopsy in this study versus surgical specimen after gastrectomy in the previous study (21). In fact, according to the previous report, only $53.3 \%(8 / 15)$ of PCC cases were correctly diagnosed as PCC on preoperative endoscopic biopsy, and the remaining $46.7 \%(7 / 15)$ of PCC cases diagnosed using surgical specimen(s) were misdiagnosed as adenocarcinoma in preoperative endoscopic biopsy (21). Therefore, there would be some limitations in the diagnosis of PCC using endoscopic biopsy, which could explain the difference in results regarding perfusion parameters of AGC between the current study and previous studies (21).

Although PCT has emerged as an attractive functional imaging modality in oncologic fields, there have been continuous challenges to acquiring perfusion parameters for the gastrointestinal tract, including the stomach, such as motion-related artifacts during PCT (21). The potential increase in radiation exposure during PCT has been another major drawback that has prevented the widespread use of PCT. In our study, we used $80 \mathrm{kVp}$ with $100 \mathrm{mAs}$, instead of the standard $120 \mathrm{kVp}$ to reduce the radiation dose of PCT. Furthermore, the scan range of PCT was meticulously adjusted to the location of the lesion, which was demonstrated on precontrast CT scan and endoscopic images for further reduction of PCT radiation dose. Consequently, the mean effective radiation dose of PCT in this study was $\mathbf{1 4 . 6}$ $\mathrm{mSv}$, which is not much higher than the routine abdominal multiphasic CT dose of 10-15 mSv, probably due to the use of low tube voltage and tight adjustment of scan range.

There are several limitations to this study that should be acknowledged. First, owing to the relatively small study population, our statistical power was not strong. Furthermore, because our investigation was a singlecenter study, selection bias may have been introduced. Therefore, to confirm the results of our study and to establish the exact role of PCT in the management of AGC patients, further multicenter studies with a larger number of patients are strongly warranted. Second, only one available software program from one specific vendor was used in our study. According to the results of several previous studies, however, there may be some discordance in perfusion parameters obtained from different software programs and, therefore, the results from different software programs may not be interchangeable (30-32). Therefore, to generalize our study results, further studies using different software programs from different vendors using various perfusion models are strongly warranted. Third, because we did not perform follow-up PCT examinations after palliative chemotherapy for unresectable AGC, we could not assess changes in perfusion parameters, especially PS, after the cycles of palliative chemotherapy. In fact, we believe that the assessment of changes in perfusion parameters of AGC during or after chemotherapy would be an interesting and important topic. Therefore, further studies including both baseline and follow-up PCT examinations after the chemotherapy cycles would be needed to address this issue.

In conclusion, perfusion parameter data acquired by PCT may be used as predictive imaging biomarkers for the evaluation of treatment response to systemic chemotherapy, given that the PS value in the responder group was significantly higher than that of non-responders.

\section{Conflicts of Interest}

The authors have no potential conflicts of interest to disclose.

\section{ORCID iDs}

Se Hyung Kim

https://orcid.org/0000-0001-8664-0356

Dong Ho Lee

https://orcid.org/0000-0001-8983-851X

Sang Min Lee

https://orcid.org/0000-0001-7719-3849

Joon Koo Han

https://orcid.org/0000-0001-5916-5545

\section{REFERENCES}

1. Parkin DM, Bray F, Ferlay J, Pisani P. Global cancer statistics, 2002. CA Cancer J Clin 2005;55:74-108

2. Soerjomataram I, Lortet-Tieulent J, Parkin DM, Ferlay J, Mathers C, Forman D, et al. Global burden of cancer in 2008: a systematic analysis of disability-adjusted life-years in 12 world regions. Lancet 2012;380:1840-1850

3. Lee DH, Kim SH, Joo I, Hur BY, Han JK. Comparison between 18F-FDG PET/MRI and MDCT for the assessment of 
preoperative staging and resectability of gastric cancer. Eur J Radiol 2016;85:1085-1091

4. Glimelius B, Ekström K, Hoffman K, Graf W, Sjödén PO, Haglund $\mathrm{U}$, et al. Randomized comparison between chemotherapy plus best supportive care with best supportive care in advanced gastric cancer. Ann Oncol 1997;8:163-168

5. Wöhrer SS, Raderer M, Hejna M. Palliative chemotherapy for advanced gastric cancer. Ann Oncol 2004;15:1585-1595

6. Eisenhauer EA, Therasse P, Bogaerts J, Schwartz LH, Sargent $D$, Ford R, et al. New response evaluation criteria in solid tumours: revised RECIST guideline (version 1.1). Eur J Cancer 2009;45:228-247

7. Koh TS, Thng CH, Lee PS, Hartono S, Rumpel H, Goh BC, et al. Hepatic metastases: in vivo assessment of perfusion parameters at dynamic contrast-enhanced MR imaging with dual-input two-compartment tracer kinetics model. Radiology 2008;249:307-320

8. Harry VN, Semple SI, Parkin DE, Gilbert FJ. Use of new imaging techniques to predict tumour response to therapy. Lancet Oncol 2010;11:92-102

9. Chung HH, Kim JW, Han KH, Eo JS, Kang KW, Park NH, et al. Prognostic value of metabolic tumor volume measured by FDG-PET/CT in patients with cervical cancer. Gynecol Oncol 2011;120:270-274

10. Kim JW, Jeong YY, Chang NK, Heo SH, Shin SS, Lee JH, et al. Perfusion CT in colorectal cancer: comparison of perfusion parameters with tumor grade and microvessel density. Korean J Radiol 2012;130:S89-S97

11. Lv WF, Han JK, Cheng DL, Zhou CZ, Ni M, Lu D. CT perfusion imaging can predict patients' survival and early response to transarterial chemo-lipiodol infusion for liver metastases from colorectal cancers. Korean J Radiol 2015;16:810-820

12. Lee DH, Kim SH, Im SA, Oh DY, Kim TY, Han JK. Multiparametric fully-integrated 18-FDG PET/MRI of advanced gastric cancer for prediction of chemotherapy response: a preliminary study. Eur Radiol 2016;26:2771-2778

13. Miles KA, Griffiths MR. Perfusion CT: a worthwhile enhancement? Br J Radiol 2003;76:220-231

14. Yi CA, Lee KS, Kim EA, Han J, Kim H, Kwon 0J, et al. Solitary pulmonary nodules: dynamic enhanced multi-detector row CT study and comparison with vascular endothelial growth factor and microvessel density. Radiology 2004;233:191-199

15. Meijerink MR, van Waesberghe JH, van der Weide L, van den Tol P, Meijer S, van Kuijk C. Total-liver-volume perfusion CT using 3-D image fusion to improve detection and characterization of liver metastases. Eur Radiol 2008:2345-2354

16. Chen TW, Yang ZG, Li Y, Li ZL, Yao J, Sun JY. Quantitative assessment of first-pass perfusion of oesophageal squamous cell carcinoma using 64-section MDCT: initial observation. Clin Radiol 2009;64:38-45

17. Zhang H, Pan Z, Du L, Yan C, Ding B, Song Q, et al. Advanced gastric cancer and perfusion imaging using a multidetector row computed tomography: correlation with prognostic determinants. Korean J Radiol 2008;9:119-127

18. Satoh A, Shuto K, Okazumi S, Ohira G, Natsume T, Hayano K, et al. Role of perfusion $\mathrm{CT}$ in assessing tumor blood flow and malignancy level of gastric cancer. Dig Surg 2010;27:253-260

19. Yao J, Yang ZG, Chen TW, Li Y, Yang L. Perfusion changes in gastric adenocarcinoma: evaluation with 64-section MDCT. Abdom Imaging 2010;35:195-202

20. Yao J, Yang ZG, Chen HJ, Chen TW, Huang J. Gastric adenocarcinoma: can perfusion $C T$ help to noninvasively evaluate tumor angiogenesis? Abdom Imaging 2011;36:15-21

21. Lee DH, Kim SH, Joo I, Han JK. CT Perfusion evaluation of gastric cancer: correlation with histologic type. Eur Radiol 2018;28:487-495

22. Kruk-Bachonko J, Krupski W, Czechowski M, Kurys-Denis E, Mądro P, Sierocińska-Sawa J, et al. Perfusion CT - A novel quantitative and qualitative imaging biomarker in gastric cancer. Eur J Radiol 2017;95:399-408

23. Shin CI, Kim SH, Lee ES, Lee DH, Hwang EJ, Chung SY, et al. Ultra-low peak voltage CT colonography: effect of iterative reconstruction algorithms on performance of radiologists who use anthropomorphic colonic phantoms. Radiology 2014;273:759-771

24. Lundsgaard Hansen M, Fallentin E, Lauridsen C, Law I, Federspiel B, Bæksgaard L, et al. Computed tomography (CT) perfusion as an early predictive marker for treatment response to neoadjuvant chemotherapy in gastroesophageal junction cancer and gastric cancer--a prospective study. PLoS One 2014;9:e97605

25. Brix G, Bahner ML, Hoffmann U, Horvath A, Schreiber W. Regional blood flow, capillary permeability, and compartmental volumes: measurement with dynamic CT-initial experience. Radiology 1999;210:269-276

26. Raghunand N, Gatenby RA, Gillies RJ. Microenvironmental and cellular consequences of altered blood flow in tumours. $\mathrm{Br} \mathrm{J}$ Radiol 2003;76:S11-S22

27. Bellomi M, Petralia G, Sonzogni A, Zampino MG, Rocca A. CT perfusion for the monitoring of neoadjuvant chemotherapy and radiation therapy in rectal carcinoma: initial experience. Radiology 2007;244:486-493

28. Cooper RA, Carrington BM, Loncaster JA, Todd SM, Davidson $\mathrm{SE}$, Logue JP, et al. Tumour oxygenation levels correlate with dynamic contrast-enhanced magnetic resonance imaging parameters in carcinoma of the cervix. Radiother Oncol 2000;57:53-59

29. Lim JS, Kim D, Baek SE, Myoung S, Choi J, Shin SJ, et al. Perfusion MRI for the prediction of treatment response after preoperative chemoradiotherapy in locally advanced rectal cancer. Eur Radiol 2012;22:1693-1700

30. Goh V, Halligan S, Bartram CI. Quantitative tumor perfusion assessment with multidetector $\mathrm{CT}$ : are measurements from two commercial software packages interchangeable? Radiology 2007;242:777-782

31. Kudo K, Sasaki M, Yamada K, Momoshima S, Utsunomiya H, 
Lee et al.

Shirato $\mathrm{H}$, et al. Differences in CT perfusion maps generated by different commercial software: quantitative analysis by using identical source data of acute stroke patients. Radiology 2010;254:200-209
32. Kim SH, Kamaya A, Willmann JK. CT perfusion of the liver: principles and applications in oncology. Radiology 2014;272:322-344 